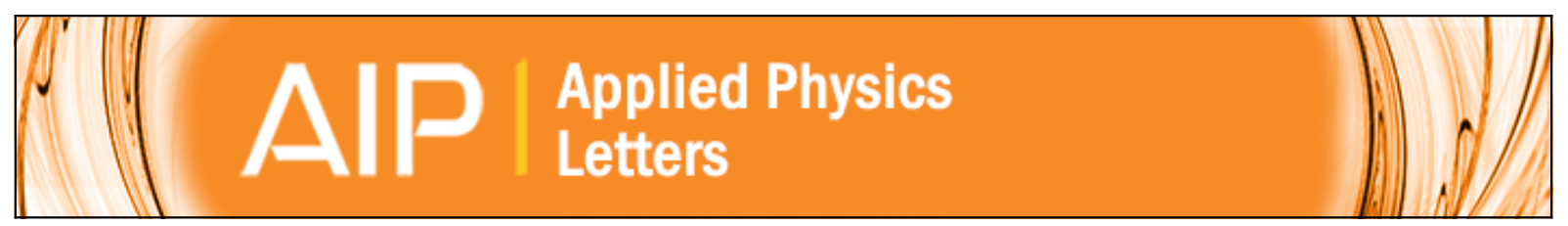

\title{
Quantitative mapping of fast voltage pulses in tunnel junctions by plasmonic luminescence
}

Christoph Grosse, Markus Etzkorn, Klaus Kuhnke, Sebastian Loth, and Klaus Kern

Citation: Applied Physics Letters 103, 183108 (2013); doi: 10.1063/1.4827556

View online: http://dx.doi.org/10.1063/1.4827556

View Table of Contents: http://scitation.aip.org/content/aip/journal/apl/103/18?ver=pdfcov

Published by the AIP Publishing

\section{Articles you may be interested in}

Resonant plasmonic terahertz detection in vertical graphene-base hot-electron transistors

J. Appl. Phys. 118, 204501 (2015); 10.1063/1.4936265

Electron tunnelling through single azurin molecules can be on/off switched by voltage pulses

Appl. Phys. Lett. 106, 183701 (2015); 10.1063/1.4919911

Fabrication of silver tips for scanning tunneling microscope induced luminescence

Rev. Sci. Instrum. 82, 083101 (2011); 10.1063/1.3617456

Direct injection tunnel spectroscopy of a $\mathrm{p}-\mathrm{n}$ junction

Appl. Phys. Lett. 95, 022106 (2009); 10.1063/1.3177191

Electric double layer effect on observable characteristics of the tunnel current through a bridged electrochemical contact

J. Chem. Phys. 127, 104708 (2007); 10.1063/1.2766954

\section{Precise temperature control for cryogenic research}

\section{Model 372}

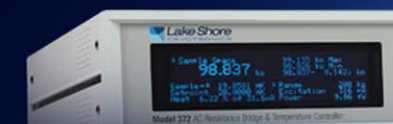




\title{
Quantitative mapping of fast voltage pulses in tunnel junctions by plasmonic luminescence
}

\author{
Christoph Grosse, ${ }^{1}$ Markus Etzkorn, ${ }^{1, a)}$ Klaus Kuhnke, ${ }^{1}$ Sebastian Loth, ${ }^{1,2, a)}$ \\ and Klaus Kern ${ }^{1,3}$ \\ ${ }^{1}$ Max Planck Institute for Solid State Research, Heisenbergstr. 1, 70569 Stuttgart, Germany \\ ${ }^{2}$ Max Planck Research Group - Dynamics of Nanoelectronic Systems, Center for Free-Electron Laser Science, \\ Luruper Chaussee 149, 22761 Hamburg, Germany \\ ${ }^{3}$ École Polytechnique Fédérale de Lausanne, Institute of Condensed Matter Physics, Bâtiment PH, Station 3, \\ 1015 Lausanne, Switzerland
}

(Received 19 September 2013; accepted 15 October 2013; published online 30 October 2013)

\begin{abstract}
An optical read-out technique is demonstrated that enables mapping the time-dependent electrostatic potential in the tunnel junction of a scanning tunneling microscope with millivolt and nanosecond accuracy. We measure the time-dependent intensity of plasmonic light emitted from the tunnel junction upon excitation with a nanosecond voltage pulse. The light intensity is found to be a quantitative measure of the voltage between tip and sample. This permits non-invasive mapping of fast voltage transients directly at the tunnel junction. Knowledge of the pulse profile reaching the tunnel junction is applied to optimize the experiment's time response by actively shaping the incident pulses. (C) 2013 AIP Publishing LLC. [http://dx.doi.org/10.1063/1.4827556]
\end{abstract}

Scanning probe microscope studies focus increasingly on measuring fast time-dependent processes at atomic dimensions, such as $\operatorname{spin}^{1-4}$ and charge ${ }^{5,6}$ dynamics or molecular motion. ${ }^{7-10}$ Heterodyne frequency mixing ${ }^{11}$ and pump-probe excitation schemes ${ }^{2,12-15}$ have been put forward as techniques to measure repetitive variations of the tunnel current in a scanning tunneling microscope (STM). Such techniques overcome practical limitations caused by the small bandwidth of high-gain current amplifiers and circumvent shot noise problems that arise in fast measurements of small electric currents. One essential prerequisite to quantitatively analyze such measurements is the exact knowledge of the time-dependent bias voltage at the tunnel junction, $V_{\mathrm{T}}(t)$. For nanosecond pulses, absorption and reflection in the cabling and connectors will significantly distort the input pulses, $V_{0}(t)$, as they propagate to the tunnel junction. Due to its enclosure inside an experimental setup, generally, the voltage at the point of the measurement, here the STM tunnel junction, is not directly accessible.

Here, we introduce a concept to measure fast variations of the electrostatic potential in a tunneling experiment. We use the voltage dependence of light emission in a metallic STM tunnel junction to measure the time-dependent voltage profile at the tunnel junction, $V_{\mathrm{T}}(t)$ (Figure 1(a)). The photons result from the decay of tip-induced plasmon polaritons which are excited by inelastically tunneling electrons. ${ }^{16}$ Plasmonic light emission from STM tunnel junctions has previously been used as a measure of noise density of point contacts. ${ }^{17}$ Plasmons at surfaces and on clusters have typical life times on the femtosecond time scale. ${ }^{18,19}$ Hence, the photons emitted from gap plasmons in the cavity between an STM tip and a metal surface may be used as an instantaneous probe for processes down to at least the picosecond range.

\footnotetext{
a) Authors to whom correspondence should be addressed. Electronic addresses: m.etzkorn@fkf.mpg.de, Tel.: +49 (0)711 689-1538 and sebastian.loth@mpsd.cfel.de, Tel.: +49 (0)40 8998-6273.
}

The experiments are performed on clean, atomically flat terraces of a $\mathrm{Ag}(111)$ single crystal in a home-built low-temperature $(5 \mathrm{~K})$ scanning tunneling microscope. To enhance plasmonic emission, the STM tips are prepared by evaporating silver onto electrochemically etched Au tips. An adjustable lens in the STM feeds the light from the tunnel junction through pinholes in the thermal shields ${ }^{20}$ to an avalanche single photon counting diode (APD, Perkin Elmer SPCMAQRH-15) outside the vacuum chamber. A continuous train of short voltage pulses is produced by pulse pattern and arbitrary waveform generators (Agilent 81110A, Figs. 1 and 2; Agilent $81150 \mathrm{~A}$, Fig. 3). The pulses are coupled into the STM by using standard low-temperature coaxial wiring. ${ }^{21}$ We record the time-dependent luminescence intensity resulting from the voltage pulses at the tunnel junction, $V_{\mathrm{T}}(t)$, with a time-correlated single photon counter (TCSPC, Becker \& Hickl SPC-130). It measures the time difference, $\Delta t$, between a trigger pulse (start) of the pulse generator and the next detected photon (stop). By counting many of these start-stop events (typically $10^{8}$ for an integration time of $500 \mathrm{~s}$ ) into time bins of the TCSPC, we obtain a histogram $N(\Delta t)$ of photon arrival times. $N(\Delta t)$ is proportional to the time-dependent luminescence intensity, $I(t)$, because the number of detection events is kept much smaller than the number of trigger pulses. The achievable experimental time resolution is limited by the time jitter of the photon detector and estimated to be less than $1.1 \mathrm{~ns}$ for the APD used here. ${ }^{22}$

The only requirement to use the photon intensity as a voltage probe is its strictly monotonic dependence on the applied voltage, since the photon emission is instantaneous on our measurement time scale (see above). Once known, this dependence can be used to convert the photon intensity back to an instantaneous transient voltage in the junction. Indeed, we find that this criterion is fulfilled for bias voltages larger than the energy threshold of the APD, about $>1.2 \mathrm{eV}$ (Fig. 1(b)). The monotonic behavior arises mostly due to the strong decrease of the electron tunnel barrier with applied 

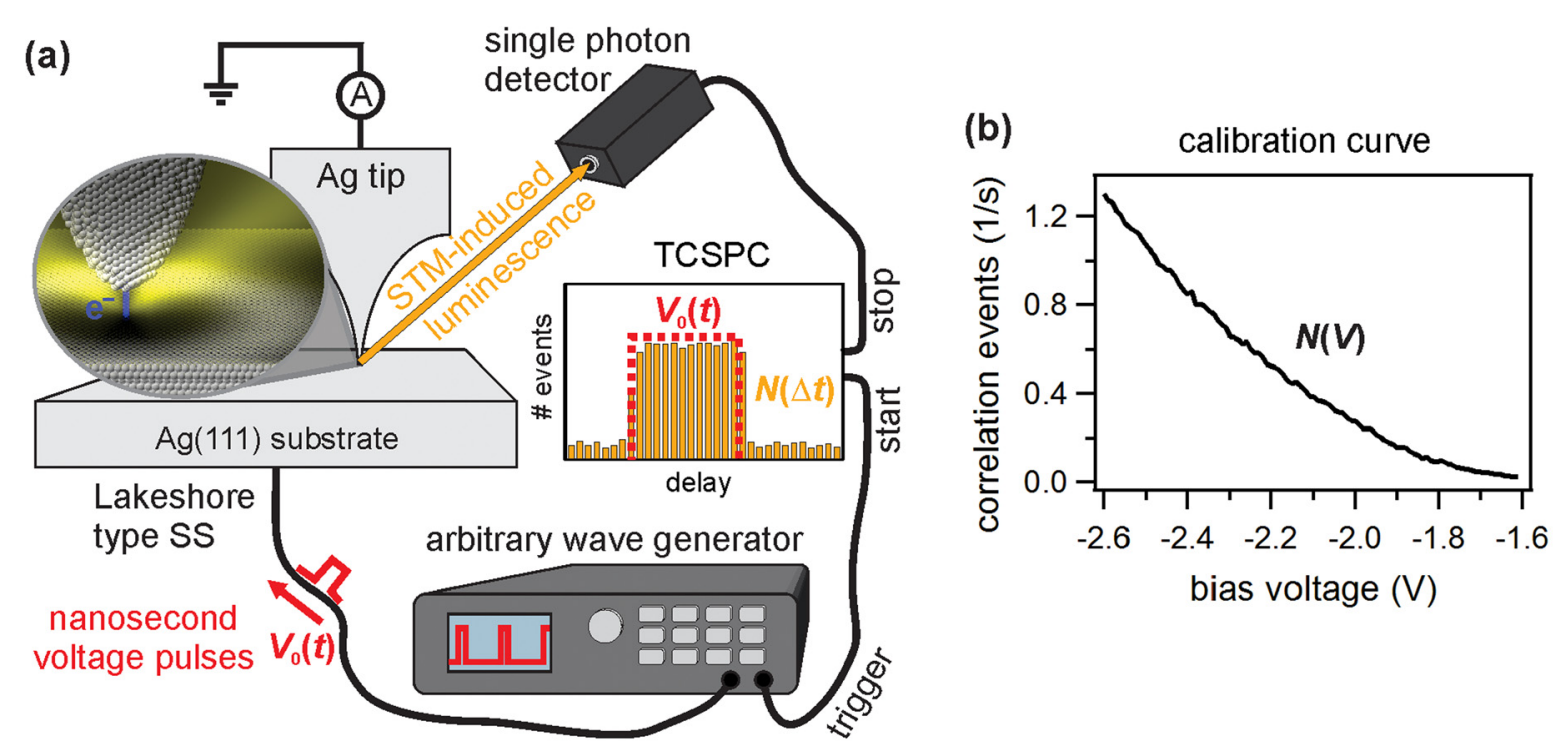

(c)
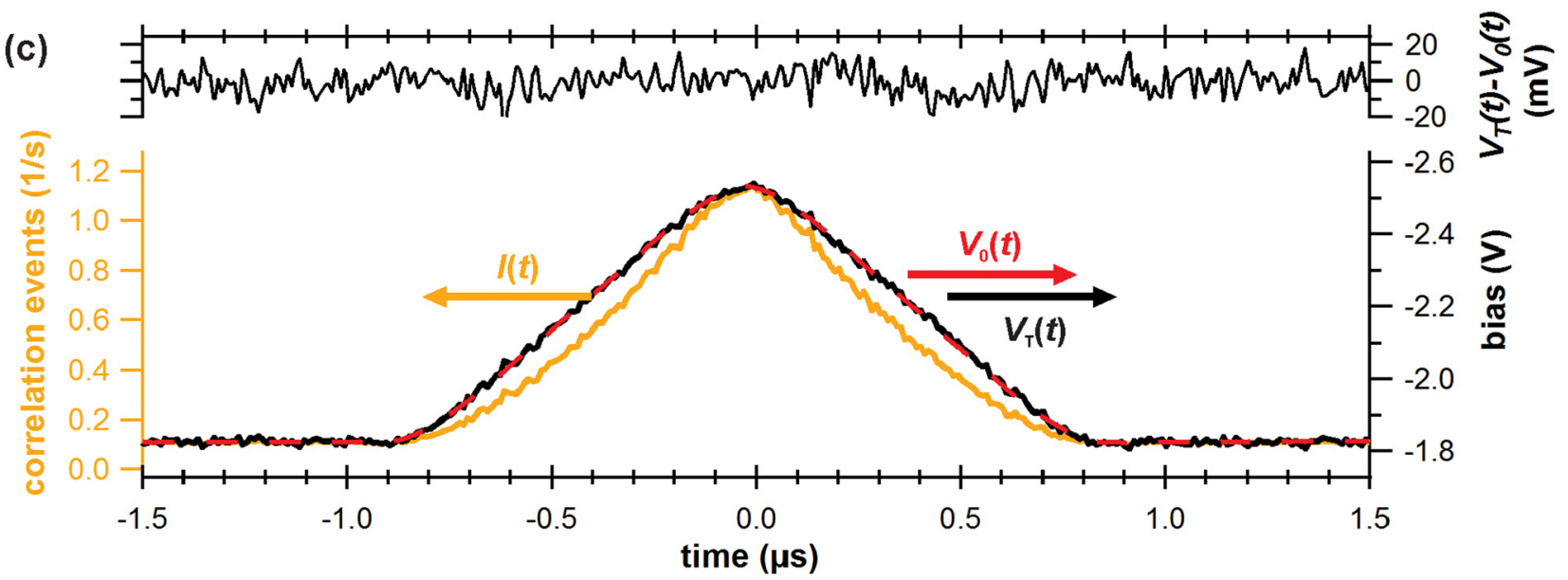

FIG. 1. (a) Schematic of the time correlating single photon counting (TCSPC) experiment. (b) Number of correlation events per bin as a function of DC bias voltage. (c) Lower graph: Voltage pulse sent to the STM $V_{0}(t)$ with a FWHM 870 ns (dashed red curve); distribution of photon arrival times (orange); and voltage profile at the tunnel junction, $V_{\mathrm{T}}(t)$ (black curve, derived from the photon signal). Upper graph: Difference between the pulse arriving at the tunnel junction and the pulse sent to the STM, $V_{\mathrm{T}}(t)-V_{0}(t)$. TCSPC bin width: $10 \mathrm{~ns}$.

voltage. Details of the functional dependence, however, may vary between different tip geometries. We thus use individual calibration curves for each set of measurements to ensure a quantitative evaluation.

To check the validity of the proposed mechanism, we send microsecond long bias pulses to the STM. For these slow pulses we expect that the voltage at the tunnel junction follows precisely the pulse shape produced by the pulse generator, that is $V_{\mathrm{T}}(t)=V_{0}(t)$. Figure 1(c) shows the measured distribution of photon arrival times, $N(\Delta t)$, (orange) recorded for a triangular voltage pulse with a full width at half maximum (FWHM) of $870 \mathrm{~ns}, V_{0}(t)$ (dashed red curve). The transient voltage $V_{\mathrm{T}}(t)$ (solid black curve) is calculated from $N(\Delta t)$ using the calibration curve in Fig. 1(b). As is apparent in the difference signal of the transient voltage and the input pulse, $V_{\mathrm{T}}(t)-V_{0}(t)$ (black curve, upper graph), the determined curve $V_{\mathrm{T}}(t)$ perfectly agrees with $V_{0}(t)$. For this measurement the error in the optically measured voltage profile is given mainly by the statistical (Poisson) noise in the measured photon signal, $N(\Delta t)$, and the accuracy of the calibration curve $N(V)$. From the root mean square of the difference $V_{\mathrm{T}}(t)-V_{0}(t)$, we obtain the voltage at the STM junction with a precision of better than $6 \mathrm{mV}$, corresponding to $<0.4 \%$ of the applied voltage.

In a next step we inject fast triangular voltage pulses (Fig. 2(a)). We find that the voltage transients at the tunnel junction deviate significantly from the injected pulses (dashed lines) already at a pulse width of $50 \mathrm{~ns}$. For even shorter pulses, we observe persistent and strong voltage oscillations extending to $>300 \mathrm{~ns}$ and showing amplitudes up to $20 \%$ of the pulse height. In order to verify that these oscillations are variations of the tunnel junction voltage, we measure the pulse autocorrelation using only STM electronics: ${ }^{12}$ two identical voltage pulses are added by a power combiner and injected into the bias line of the STM. The delay, $\Delta \tau$, between the two pulses is scanned and the resulting time averaged tunnel current $\langle J(\Delta \tau)\rangle$ is recorded as a function of $\Delta \tau$. We apply the same pulses as in Fig. 2(a), but chose the amplitude such that the bias varies over a range in which the tunnel current $J\left(V_{\mathrm{T}}\right)$ is nonlinear. Due to the rectifying property of this nonlinearity, the time-averaged tunnel current changes when parts of the pulses overlap in time. The all-electronic autocorrelation measurement (Fig. 2(b)) also shows strong ringing. We calculate the autocorrelation 

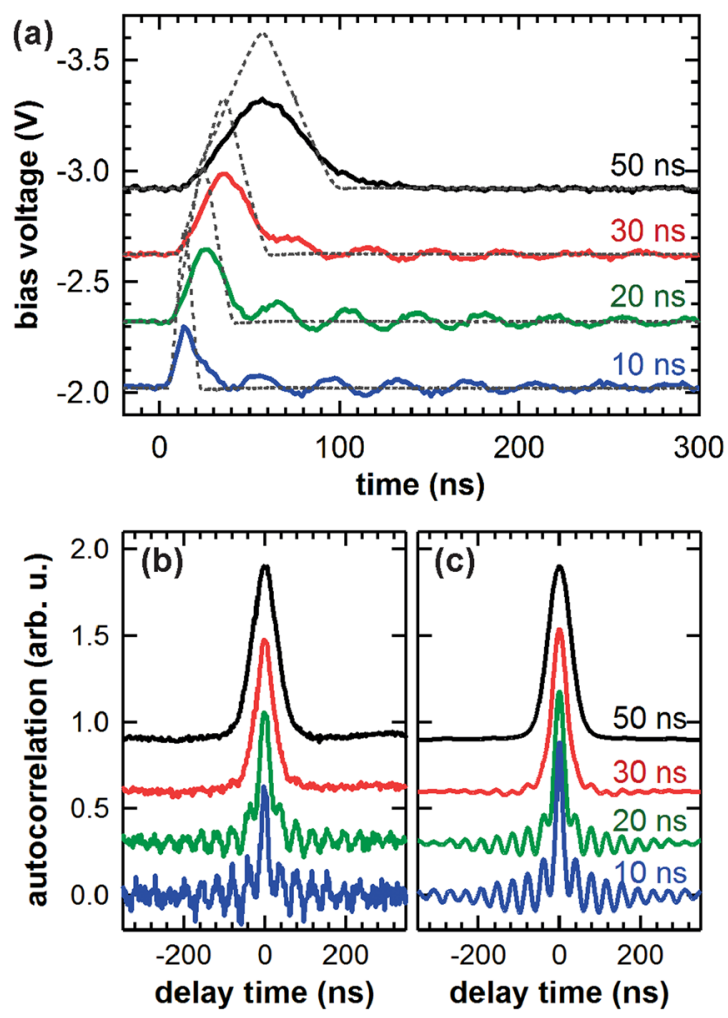

FIG. 2. (a) Optically measured voltage profiles at the tunnel junction (colored curves) for fast triangular pulses of different widths sent to the STM (dashed curves, pulse widths denoted). All curves were recorded at $-2.02 \mathrm{~V}$ offset and are shifted for clarity by increments of $0.3 \mathrm{~V}$. TCSCP bin width: 2 ns. (b) Electronically measured autocorrelation using the same (colorcoded) pulses as in (a). (c) Numerically derived autocorrelation of the optically measured pulses in (a).

of the optically measured voltage transients (Fig. 2(c)) and find excellent agreement with the measured pulse autocorrelation. This confirms the reliability of the optical approach to measure fast voltage transients.

Fig. 3(a) shows transient voltages $V_{\mathrm{T}}(t)$ for fast rectangular-shaped pulses of different length. The pulses of the function generator have a $2 \mathrm{~ns}$ falling edge, whereas the pulses arriving at the tunnel junction are found to have falling edges of approximately $50 \mathrm{~ns}$ width (90\%-10\% amplitude). This is most likely due to the finite bandwidth of the coaxial cables of $20 \mathrm{MHz}{ }^{21}$ Again, ringing is observed. Such strong deviations from the injected pulses are undesired and can obscure the dynamics in any time-resolved STM measurement. In order to quantify the transmission between pulse generator and tunnel junction, we determine the transfer function $T(v)$ as the ratio of the Fourier transforms of $V_{\mathrm{T}}(t)$ and $V_{0}(t)$, Fig. 3(b) (black curves). Alternatively, the transfer function is accessible by using only electronics (Fig. 3(b), green curve), analogous to the pulse autocorrelation discussed above, by adding a sine-wave voltage to a DC bias and using the same nonlinearity as for the all-electronic autocorrelation.

From the two independent measurements of the transfer function, we find that the ringing can be traced to three strong resonances at $25 \mathrm{MHz}, 90 \mathrm{MHz}$, and $130 \mathrm{MHz}$. They most likely result from built-up of standing waves between the high-impedance tunnel junction and impedance jumps at intersections between different wire segments along the transmission line. It is worth noting that similar resonances and low passing behavior are likely to occur in any STM system that does not use special high-frequency wiring. Use of high-frequency connectors and wires can reduce such resonances, ${ }^{13,14}$ but may not always be implementable in commercial or existing STMs, in which vibration isolation, ultra-high vacuum environment, low temperatures or high magnetic fields may limit the choice of high-frequency components.

With knowledge of the amplitude and phase of the transfer function, however, it is possible to circumvent imperfections of the experimental setup by a proper choice of the input pulses. We generate a voltage profile, $V_{0}(t)$, shaped such that after propagating through the imperfect wire it reaches the tunneling junction with the desired profile. This method is akin to pulse shaping in optical experiments ${ }^{23}$ or pulse optimization in the transmission of digital signals. $^{24}$ Fig. 3(c) shows the modified input voltage profiles obtained by multiplying the frequency components of the desired rectangular pulse shape with the inverse transfer function $T^{-1}(v)$. After propagation through the transmission line, these pulses lead to near-perfect rectangular pulses with significantly reduced ringing and falling edges of $3 \mathrm{~ns}$ (90\%-10\%) width (Fig. 3(d)). Residual voltage variations visible in Fig. 3(d) arise partly from noise in the transfer function which is calculated from data (Fig. 3(a)) and thus itself contains noise. In total, we achieve a factor 16 increase in performance and time response of the STM without physical modification to the wiring. This pulse shaping method is generally applicable and allows correction of low-passing behavior and ringing in the bias line.

The experimental limit with respect to the compensation of cable imperfections will be given by the bandwidth and the maximum output amplitude of the arbitrary waveform generator and may reach well below 1 ns. For cables with good high frequency performance, switching times will be limited by parasitic capacitances of the STM head and voltage pulses as fast as 8 ps have been demonstrated. ${ }^{12}$ With respect to the optical detection scheme, the limit of time resolution will be determined by the time jitter of the employed photon counting detector. The ultimate limit is, finally, defined by the plasmon lifetime, which is in the subpicosecond range.

In summary, we demonstrate a method to map the profile of nanosecond short voltage pulses sent to the tunnel junction of a scanning tunneling microscope with $\mathrm{mV}$ accuracy. By using the monotonic dependence of the plasmon emission on metal surfaces on the applied bias voltage, the time-dependent voltage at the junction $V_{\mathrm{T}}(\mathrm{t})$ can be determined directly from the time-dependent intensity. Quantitative knowledge of the transient voltage at the tunnel junction can be used to optimize the signal quality in the tunnel junction significantly. In this experiment, we achieved a time resolution of $3 \mathrm{~ns}$, exceeding the bandwidth of the wiring more than one order of magnitude.

The presented technique is non-invasive and can be used in any tunneling transport experiment in which plasmon-induced light emission is detectable. It may be applied to measure fast changes in the local electrostatic potential at the tunnel junction as caused by non-equilibrium 

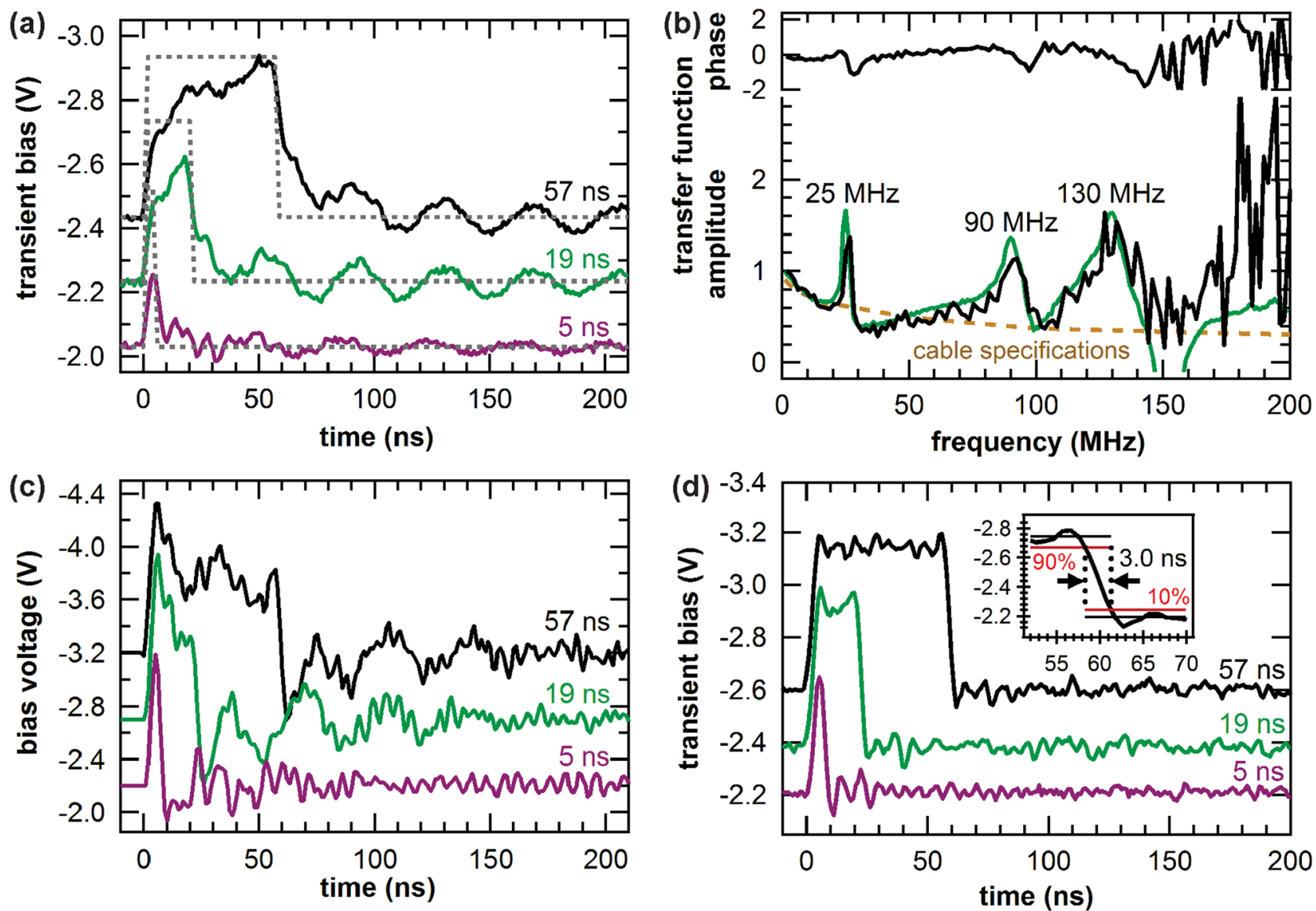

FIG. 3. (a) Optically measured transient voltages (colored curves) for rectangular pulses of different duration sent to the STM (dashed curves, pulse width denoted). (b) Transfer function of the transmission line between generator and tunnel junction. Black curves: Amplitude (normalized to 1 at $0 \mathrm{MHz}$ ) and phase (in radians) derived from the optically recorded data in (a); green curve: measured on a nonlinear current-bias characteristic; brown dashed curve: cable specifications, see Ref. 20. (c) Shaped voltage pulses sent to the STM to compensate for the distortion by the cables. (d) Optically measured transients for the pulses in (c). The inset in (d) shows a zoom in on the falling edge of the $57 \mathrm{~ns}$ pulse. TCSPC bin width in (a), (c), and (d): $1 \mathrm{~ns}$. All curves in (a) were measured at an offset of $-2.03 \mathrm{~V}$ and the curves in (b) and (c) at an offset of $-2.2 \mathrm{~V}$. Curves shifted for clarity by multiples of $0.2 \mathrm{~V}$ in (a) and (d) and $0.5 \mathrm{~V}$ in (c).

effects such as fluctuating charges on dopants or molecules, or transient screening by dipoles. Applied in STM, the generation of precisely defined, sharp voltage pulses at the tunnel junctions will provide the foundation to study luminescence dynamics, that is the formation and recombination of electron-hole pairs, on nanometer length scales.

${ }^{1}$ S. Krause, L. Berbil-Bautista, G. Herzog, M. Bode, and R. Wiesendanger, Science 317(5844), 1537 (2007).

${ }^{2}$ S. Loth, M. Etzkorn, C. P. Lutz, D. M. Eigler, and A. J. Heinrich, Science 329(5999), 1628 (2010).

${ }^{3}$ S. Loth, S. Baumann, C. P. Lutz, D. M. Eigler, and A. Heinrich, Science 335(6065), 196 (2012).

${ }^{4}$ A. A. Khajetoorians, B. Baxevanis, C. Huebner, T. Schlenk, S. Krause, T. O. Wehling, S. Lounis, A. Lichtenstein, D. Pfannkuche, J. Wiebe, and R. Wiesendanger, Science 339(6115), 55 (2013).

${ }^{5}$ S. Yoshida, M. Yokota, O. Takeuchi, H. Oigawa, Y. Mera, and H. Shigekawa, Appl. Phys. Express 6(3), 032401 (2013).

${ }^{6}$ Y. Terada, S. Yoshida, O. Takeuchi, and H. Shigekawa, Nature Photon. 4(12), 869 (2010).

${ }^{7}$ Y. Sainoo, Y. Kim, T. Okawa, T. Komeda, H. Shigekawa, and M. Kawai, Phys. Rev. Lett. 95(24), 246102 (2005).

${ }^{8}$ M. Mehlhorn, H. Gawronski, and K. Morgenstern, Phys. Rev. Lett. 104(7), 076101 (2010).

${ }^{9}$ T. Kumagai, A. Shiotari, H. Okuyama, S. Hatta, T. Aruga, I. Hamada, T. Frederiksen, and H. Ueba, Nature Mater. 11(2), 167 (2012).

${ }^{10}$ A. van Houselt and H. J. W. Zandvliet, Rev. Mod. Phys. 82(2), 1593 (2010).
${ }^{11}$ M. R. Kan, D. C. Fortin, E. Finley, K. M. Cheng, M. R. Freeman, and W. K. Hiebert, Appl. Phys. Lett. 97(25), 253108 (2010).

${ }^{12}$ G. M. Steeves, A. Y. Elezzabi, R. Teshima, R. A. Said, and M. R. Freeman, IEEE J. Quantum Electron. 34(8), 1415 (1998).

${ }^{13}$ C. Saunus, J. R. Bindel, M. Pratzer, and M. Morgenstern, Appl. Phys. Lett. 102(5), 051601 (2013).

${ }^{14}$ I. Moult, M. Herve, and Y. Pennec, Appl. Phys. Lett. 98(23), 233103 (2011).

${ }^{15}$ T. L. Cocker, V. J. Jelic, M. Gupta, S. J. Molesky, J. A. J. Burgess, G. De Los Reyes, L. V. Titova, Y. Y. Tsui, M. R. Freeman, and F. A. Hegmann, Nature Photon. 7, 620 (2013).

${ }^{16}$ J. K. Gimzewski, J. K. Sass, R. R. Schlitter, and J. Schott, Europhys. Lett. 8(5), 435 (1989).

${ }^{17}$ N. L. Schneider, G. Schull, and R. Berndt, Phys. Rev. Lett. 105(2), 026601 (2010).

${ }^{18}$ U. D. Keil, T. Ha, J. R. Jensen, and J. M. Hvam, Appl. Phys. Lett. 72(23), 3074 (1998).

${ }^{19}$ D. Steinmüller-Nethl, R. A. Höpfel, E. Gornik, A. Leitner, and F. R. Aussenegg, Phys. Rev. Lett. 68(3), 389 (1992).

${ }^{20}$ K. Kuhnke, A. Kabakchiev, W. Stiepany, F. Zinser, R. Vogelgesang, and K. Kern, Rev. Sci. Instrum. 81(11), 113102 (2010).

${ }^{21}$ Lake Shore Cryotronics Inc., Stainless steel cable type SS, length $1.5 \mathrm{~m}$, attenuation $-3 \mathrm{~dB} / \mathrm{m}$ at approximately $20 \mathrm{MHz}, 2013$.

${ }^{22}$ K. Kuhnke, A. Kabakchiev, T. Lutz, and K. Kern, Phys. Status Solidi B 249(4), 644 (2012).

${ }^{23}$ A. M. Weiner, J. P. Heritage, and E. M. Kirschner, J. Opt. Soc. Am. B $\mathbf{5}(8), 1563$ (1988).

${ }^{24}$ R. F. H. Fischer, Precoding and Signal Shaping for Digital Transmission (John Wiley \& Sons, New York, 2002). 